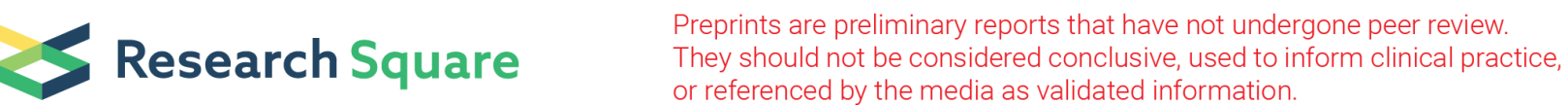

\section{Culturally Adapted Family Intervention for People With Schizophrenia in Indonesia (FUSION): A Development and Feasibility Study Protocol}

\section{Laoise Jean Renwick ( $\sim$ laoiserenwick@gmail.com )}

University of Manchester https://orcid.org/0000-0001-7060-4537

Herni Susanti

Universitas Indonesia

\section{Helen Brooks}

University of Manchester School of Medicine: The University of Manchester Faculty of Biology Medicine and Health

\section{Budi Anna Keliat}

Universitas Indonesia

\section{Tim Bradshaw}

University of Manchester School of Medicine: The University of Manchester Faculty of Biology Medicine and Health

\section{Penny Bee}

University of Manchester School of Medicine: The University of Manchester Faculty of Biology Medicine and Health

Karina Lovell

University of manchester

\section{Study Protocol}

Keywords: protocol, mixed methods, complex intervention development, feasibility study, family interventions for psychosis, caregiver burden, co-production, task-shifting, low- and middle-income countries

Posted Date: October 28th, 2021

DOI: https://doi.org/10.21203/rs.3.rs-994129/v1

License: (1) (1) This work is licensed under a Creative Commons Attribution 4.0 International License. Read Full License 


\section{Abstract \\ Background}

Mental illnesses comprise the single largest source of health-related economic burden globally and lowand middle-income countries are disproportionately affected. The majority of people with schizophrenia who need treatment do not receive it and are often wholly reliant on family caregivers for daily care and support. Family interventions have an exceptionally robust evidence base for their efficacy in high resource settings, but it is unknown whether they can produce equivalent effects in some low resource settings where cultural beliefs, explanatory models of illness and contextual socio-economic issues differ.

\section{Methods}

This protocol describes methods to culturally adapt and refine an evidence-based, family intervention for relatives and caregivers of people with schizophrenia in Indonesia. The feasibility and acceptability of implementing an adapted, co-produced intervention via task-shifting in primary care settings will be evaluated using the Medical Research Council framework for complex interventions. The development and preliminary testing of the adapted intervention will occur in three phases utilising mixed methods; stakeholder and key informant interviews will inform consensus workshops using nominal group techniques to develop a testable intervention. The feasibility of testing the intervention in a larger trial and the acceptability of the intervention will be explored in a feasibility trial of adapted family interventions compared to standard treatment. Participants in Phase 1 consultation groups will comprise service-users $(n=10-15)$, carers $(n=10-15)$ and key stakeholders including lay and healthcare workers $(n=10-15)$ and semi-structured interviews with key informants to identify implementation challenges and facilitators $(n=$ 10). Phase 2 participants will include expert stakeholders including experts by experience to achieve consensus on the essential elements of the intervention $(n=20)$ and a separate group to develop training methods and materials for our task-shifted intervention. Phase 3 will comprise 60 carer-service-user dyads participating in a randomised feasibility trial.

\section{Discussion}

National healthcare policy in Indonesia supports the delivery of mental health services in a complex network of primary care centres. This study will provide important information on the feasibility of delivering family interventions for people with schizophrenia via task-shifting in primary care settings in Indonesia.

\section{Background}

Mental illnesses comprise the single largest source of health-related economic burden globally (1) and low-and middle income countries are disproportionately affected. Schizophrenia, the most common 
psychotic illness, is among the ten most disabling conditions worldwide and global burden is projected to rise (2). High treatment gaps contribute significantly to illness burden; less than a third of those who develop schizophrenia access treatment in lower resource settings (3). In Indonesia, as in other lower resource settings, people with schizophrenia are often wholly reliant on family caregivers for daily care and support $(4,5)$. Fewer resources, poor infrastructure and a lack of trained professionals to deliver evidence-based care are prominent external factors that lead to increased caregiver burden (6). Caregivers often attribute supernatural causes to mental illnesses in Indonesia and seek care from traditional healers or shamans (4).

Effective packages of care for schizophrenia comprise both pharmacological and psychosocial interventions (7). It is broadly considered that, in Low and middle income countries, a narrower group of interventions will be feasible due to lack of finance and infrastructure, population density and underdeveloped social welfare systems (8). The World Bank's recently published third edition of global disease priorities (DCP3) includes family interventions for psychosis as one of only three potentially cost-effective interventions for people with schizophrenia and recommend these interventions should be prioritised in Low and middle income countries (9). Family interventions have an exceptionally robust evidence base for their efficacy in high resource settings $(10,11)$ and have an emerging evidence base in low and middle income countries (12). Providing these interventions can reduce relapse, and improve the family environment and therapeutic alliances with healthcare workers (13).

Family members and caregivers of patients with severe mental illness experience considerable burden (14), exceeding the burden experienced by family members caring for those with comparable long-term physical illnesses such as cancer. Families report significant psychological distress, lower quality of life and increased anxiety and depression (15). Meta-analytic studies show that interventions comprising both psychoeducation and psychotherapeutic elements can reduce the risk of relapse and rehospitalisation $(10,16)$, increase adherence to medication regimes $(10,16)$, enhance functioning $(17)$ and improve family environment $(10,18)$. Psychoeducational interventions minimise perceived burden and negative caregiving experiences (11). There is also evidence that families contribute positively to the wellbeing of people with psychosis, particularly if they themselves are supported by family interventions (16).

However, family interventions designed and tested for delivery in high-income countries, may not produce equivalent effects in low and middle income countries as they do not allow for cultural beliefs, explanatory models of illness and contextual socio-economic issues to be incorporated into intervention content and delivery (19). Indeed, when effective interventions are successfully adapted, the acceptability of interventions increases, and people are more likely to engage with help that is offered. Interventions within specific cultural groups delivered in their native language are twice as efficacious as those delivered without adaptation and cultural adaptation enhances intervention efficacy for treating schizophrenia, the degree of adaptation closely correlated with the degree of efficacy (19). 
This study describes a protocol for a mixed methods inquiry guided by an accepted framework for developing complex interventions to adapt an existing evidence-based intervention. In addition, we aim to determine whether it would be feasible to test the effectiveness of this intervention in a randomised trial evaluating recruitment, retention, and participant engagement with the intervention. Coinciding with this, we will assess healthcare workers acceptance of the intervention model and optimise sustainability by evaluating barriers and enablers from the perspectives of stakeholders and key informants in positions of potential influence to the delivery of mental healthcare locally and regionally. A third and equal priority aim, is to determine whether the intervention can be delivered via shifting this task to non-specialist healthcare workers. Task-shifted interventions for non-communicable diseases can provide cost savings without compromising on quality (20) and should be explored as an option for lower resource settings to deliver evidence-based treatments and alleviate health system inefficiencies (21).

\section{Methods}

\subsection{Aims and objectives}

This study details the methods to be used to culturally adapt and refine an evidence-based, family intervention for relatives and caregivers of people with schizophrenia in Indonesia and to evaluate the feasibility and acceptability of implementing these interventions in primary care settings. Using the Medical Research Council framework for complex interventions we will conduct a three-phase study focusing on earlier phases of development and feasibility testing (22). We will combine stakeholder consultation, synthesis, and consensus workshops using service-users, carers and healthcare professional's perspectives to develop a manual to guide intervention development. We will train healthcare workers to deliver the intervention and assess the feasibility and acceptability of conducting a randomised, single-blind trial of our co-produced, culturally relevant, evidence-based intervention to reduce relapse when compared with standard care.

The study objectives are to:

I. Adapt and refine an evidence-based family intervention using stakeholder preferences in an empirically derived heuristic framework

II. Co- produce a manual with people with schizophrenia, carers and healthcare professionals to support the delivery of the culturally relevant, adapted intervention

III. Identify training needs for non-specialist healthcare workers in primary care and train healthcare workers to deliver the intervention

IV. Evaluate the feasibility of conducting a full trial to test the effectiveness of our intervention and explore the acceptability of the intervention

\subsection{Design}


The development and preliminary testing of the adapted intervention will occur in three phases utilising mixed methods; stakeholder and key informant interviews will inform consensus workshops using nominal group techniques to develop a testable intervention. The feasibility of testing the intervention in a larger trial and the acceptability of the intervention will be explored in a randomised controlled trial of adapted family interventions compared to standard treatment.

Phase 1 comprises a series of up to 4 stakeholder consultation groups including single and mixed stakeholder groups (people with schizophrenia $n=1$, carers/relatives $n=1$, healthcare professionals $n=$ 1 , mixed stakeholders $n=1$ ) to explore attitudes and beliefs about the support needs of families, experiences of accessing services, priorities for treatment delivery, preferences for content and their views on what training should provide. The consultation content and process will be underpinned by and existing heuristic framework for intervention adaptation and a widely used, evidence-based, cognitivebehavioural model of Family interventions $(19,23)$. Individual qualitative interviews will be conducted with key informants $(n=10)$ identified as key contributors with specialist roles among service managers, commissioners, ministry officials and community leaders to explore the wider implications of intervention implementation and evaluate factors affecting reach, adoption, and maintenance of interventions in primary care settings and the charity sector.

Phase 2 comprises consensus workshops with structured synthesis of findings from phase 1 and 2 to coproduce a manual to support delivery of a culturally relevant Family interventions, and resources to support sustainable training methods for future intervention delivery. We will convene two separate workshops using an expert consensus panel comprising individuals from key professional and stakeholder groups comprising people with schizophrenia, carers and healthcare workers. We will use a modified nominal group technique to gain consensus on the intervention contents, delivery format and training needs for healthcare professionals.

Phase 3 will evaluate the feasibility and acceptability of delivering our culturally adapted Family interventions in a randomised trial. We will examine the feasibility of delivering the intervention and recruiting and service-users and family members and determine whether they are willing to be randomised, are retained in the study and willing to complete measures of outcome. We will examine the acceptability of the intervention, usability of the intervention resources and explore the training needs of non-mental health trained and mental health trained healthcare workers in primary care settings.

\subsection{Research Advisory Group}

The study is supported by a Research Advisory Group (RAG) comprising service-users, carers and advocates, healthcare professionals and primary care workers, academics, community leaders and government healthcare officials $(n=11)$. The purpose of the advisory group is to provide insight and information on the needs of the researchers and the research project. The terms of reference have been agreed. The group will provide expertise and contribution to support each successive phase of the study - examples of tasks include comment on analysis of qualitative data, comment on the presentation of intervention and training manuals once developed, comment on assessment schedules for the feasibility 
study. The RAG will also take a lead role in the dissemination phase devising Plans for investigators and sponsor to communicate trial results to participants, healthcare professionals, the public, and other relevant groups. The group will be chaired by the Director of Mental Health and Drug Control, Ministry of Health in Indonesia. Additional service-user and carer representatives will be recruited at each phase and service-user involvement organised by our charity partners Komunitas Peduli Skizofreni Indonesia (KPSI). KPSI is a user-led charity which runs peer support groups, education and anti-stigma activities in health services and local communities.

\subsection{Setting and Context}

Indonesia is a large archipelago comprising approximately 17,000 islands and roughly 300 different tribes. The prevalence of psychotic illness is 1.8 per 1,000 and there is an estimated 2.6 million people with schizophrenia (24). Mental healthcare is largely provided in one of 14 regional public hospitals. Community mental health provision is limited although there is emerging specialist mental healthcare provided within a more advanced network of primary care clinics; puskesmas and posyandu provide comprehensive healthcare for all healthcare needs at district and sub-district level and approximately. Prevailing explanatory models of mental illness favour supernatural theories over biomedical explanations but often families lack knowledge of treatment availability and approaches to recovery to manage crises and support social functioning. The United Nations Sustainable Development Goals have for the first time focused on reducing the burden of mental illness scaling up prevention and treatment strategies. Coupled with the World Health Organisations focus on task-shifting to increase capacity for intervention delivery and integration between primary and secondary mental health services, there is a need to develop family interventions that are evidence-based, can be delivered by non-specialist professionals and are scalable. Task-shifting describes when healthcare tasks are redistributed to enhance the performance of health systems. Typically, tasks normally provided by a specialist of highly trained health worker is transferred to a healthcare worker with a lower level of education and training or a person specifically trained to perform a limited task such as peer or lay workers. National healthcare policy in Indonesia supports the delivery of mental health services in primary care and aims to provide universal healthcare provision for those with diagnosed mental health conditions (25). We have received ethical approval for Phase 1 and Phase 2 and covid amendments have been approved (version 4, date 01/03/2021) which entail remote study procedures including study team communication plans and data collection.

\subsection{Recruitment}

Recruitment will take place from September 2021 for a period of 21 months. Participants comprising service-users, carers and relatives and healthcare professionals through phase 1-3 will be primarily recruited from primary health care centres in Bogor and Jakarta. We will also recruit through our NGO partners, KPSI, and we will develop a social media strategy utilising existing Facebook networks to recruit potential participants to all phases of the study. 
In phase 1 we aim to explore stakeholders' priorities and preferences for implementing Family interventions using an existing effective model and we will purposively sample people with schizophrenia and their caregivers, approximately 10-15 from each group, based on gender, age, geographical setting and service attendance. Healthcare professionals and kaders from primary care centres at district and sub-district level including non-mental health trained and mental health trained workers will be included in phase 1. We also aim to understand the wider implications of intervention implementation and evaluate factors affecting reach, adoption, and maintenance of interventions in primary care settings and the charity sector. Thus, we will recruit an additional sample in phase 1 comprising key contributors with specialist roles $(n=10)$ among service managers, commissioners, ministry officials and community leaders and will be identified using snowball sampling.

To gain consensus on the key elements and adaptations of the intervention and training needs we will recruit two groups to participate in workshops to develop the intervention and implementation resource. Group 1 will comprise service-users, carers and relatives, healthcare professionals, community leaders and charity partners $(n=20)$ and group 2 will comprise a greater proportion of academic teaching and research professionals $(n=20)$. Phase 3 recruits will consist of carer/relative and service-user dyads and informed consent will first be obtained from the service-user $(n=60,30$ per arm). (see Table 1 for inclusion and exclusion criteria)

\subsection{Data Collection}

Demographic data collected include age, gender, ethnicity, marital status, education, and employment will be collated. As appropriate, we will gather information about the living arrangements, primary diagnosis of service user, duration of the caring role, number of people cared for, relationship to the person with schizophrenia, whether they live with the person, level, and type of contact and whether they are receiving support from mental health services. Healthcare professionals will provide information about the nature of their work, whether they have received specific mental health training, contact with people with mental health problems and duration of service.

Phase 1 qualitative interviews and stakeholder consultation groups will be co-facilitated by trained researchers and study team members. Information will be provided including study background, purpose of the consultation and session content and process at commencement. Participants will be asked sequentially; experiences of accessing and attending services, perceptions of psychological therapies, impact of illness on families and carers, suggestions to enhance the cultural validity of the intervention, preference for delivery including session duration and location, therapist preference, suggestions for resources that would support delivery and expectations for therapeutic aims and outcomes (26). Qualitative interviews will be audio-taped and transcribed verbatim.

Phase 2 data will be collected during two separate workshops using an expert consensus panel comprising individuals from key professional and stakeholder groups $(n=20)$ and use a modified nominal group technique to gain consensus. The panel will be identified and appointed by the RAG. Briefing information comprising the rationale for the study and a review of the evidence matrix alongside a 
participant information sheet will be provided in advance (27). Real-time interactive software will be used to allow participants to vote on intervention components and training resources comprising the core constructs of the intervention, content and delivery format and manual contents. Additional components and resources required to accompany training will be generated during these workshops using co-design group principles developed by the King's Fund (28). Small-group discussions of 4-6 participants will be facilitated by non-participant researchers focusing on areas of disagreement and clarifying each point raised for successive ranking exercises. Detailed notes will be gathered by researchers and recorded to support manual refinement.

\subsection{Analysis and Synthesis}

Phase 1 data will be analysed using a six-stage thematic analysis framework (29) using Nvivo software to organise text and support analysis. Interviews will be transcribed and independently coded by Indonesian researchers. The coding frame will be translated and verified among the wider research and study team and additionally, a sample will be translated into English and back-translated to verify coding frames (30). We will develop an evidence matrix combining empirical findings from phase 1 and 2 of the study with empirical findings from existing evidence synthesis of cultural adaptation for psychosocial and mental health interventions. Using modified nominal group techniques in phase 2 , we will determine stakeholder's views as a group on intervention components, manual resources and training needs aggregating responses producing proportional rankings for each component. A minimum defining threshold will be set for accepting items based on the proportion of items endorsed by respondents. The manual will be developed by the wider study team defining therapeutic aims and we will develop detailed procedures, patient exercise, materials and resources, good practice examples, scripts for intervention delivery and measures of processes and outcomes.

\subsection{Synthesis and Intervention Development}

To synthesise findings from phase 1 and 2 we will draw on two recent systematic reviews; one summarising the content and adaptation process, including moderators and mediators of effective interventions, in meta-analytic studies of culturally-adapted interventions for mental health problems (31) and another meta-analysis and review of culturally-adapted psychosocial interventions specifically for schizophrenia that provides an empirically-derived heuristic framework for cultural adaptation of psychosocial interventions (19). The synthesis matrix will be developed by the study team tabulating findings from the existing heuristic framework by source of evidence focusing on points of commonality, divergence and representing key ideas about intervention content and delivery, barriers and facilitators to delivery and engagement and supplemented with points about adoption and implementation.

The intervention is based on a widely used cognitive-behavioural model of family interventions developed by Barrowclough and Tarrier (23) and adopted as the model of choice in NICE-approved interventions. The family intervention will offer therapeutic elements to enhance family skills and knowledge, communication skills training, enhancing skills in problem-solving and goal setting underpinned by cognitive models and framed by collaborative partnerships to enhance supportive therapeutic 
relationships. The intervention will be delivered over 12 weeks and will consist of up to 10 sessions. The therapist guide will comprise the manualised intervention guide and details of the intervention will be made explicit following phase 1 and 2.

\subsection{Feasibility Testing}

Over 12 months, we will recruit a convenience sample of 60 service-user and family member dyads to i) compare recruitment and retention in different settings and delivered by different health professionals, ii) assess the feasibility of collecting participant outcome measures at study entry, post-intervention and six months later, iii) assess the usability and acceptance of the intervention manual by non-specialist healthcare workers in primary care, iv) measure fidelity to the model and healthcare workers adherence to the intervention model and v) assess the acceptability of the intervention to recipients. Service-user, family member and staff outcome schedules will be refined by the RAG to include symptom severity, relapse rates and hospital episodes, social functioning, family environment and functioning, knowledge, attitudes and burden and therapeutic engagement and the existing schedule has been developed from reviewing the extant literature. See Table 2.

Service-users deemed potentially eligible by healthcare workers in primary care centres will be approached by clinical staff to participate and if interested, their information will be conveyed to the recruiting research assistant who will inform about the study, screen for eligibility, and obtain consent. Next, baseline assessments will be completed with those eligible who wish to take part. Participants will be randomised to receive family interventions or to continue with treatment as usual at a ratio of 1:1. Randomisation will be carried out remotely using randomisation software according to the International Conference on Harmonization E9 Statistical Principles Guidelines and will be implemented by the trial manager. The recruiting research assistant will conduct all subsequent assessments immediately postintervention and at 3 months. Measures implemented to blind the evaluator will include advising participants not to disclose their treatment status to researchers, conducting assessments away from the treatment setting and ensuring the research assistant collecting data is separate from the trial manager. The project will primarily use the Research Data Service at UI to store, manage and curate data. Data will be stored using word and excel documents and transferred to research analysis software when required for analysis and distribution checks. Quality checks will be conducted periodically and coding will be overseen by the study teams at UI and UoM. We will also utilise the UoM Research Data Management Service (RDMS) which provides managed, secure, replicated storage. The RDMS allows researchers to securely transfer digital data to UoM and can be used to store, manage and curate data to preserve this after the lifecycle of the project. Non-digital data e.g. consent forms and manuals generated from the research programme, will be stored in stand-alone locked cabinets held in a secure location in UI. Data will be stored in raw, processed, analysed, and final dataset format to ensure quality and will be transferred between host and sponsor university using Dropbox for Business.

\subsection{Fidelity Testing and Process Evaluation}


A checklist of items will be developed from the training manual defining core components of the intervention and a percentage of manual-specified components delivered as intended will be collated from detailed diaries recorded by researchers independent of intervention delivery. Healthcare workers delivering the intervention will keep a diary following each session to evaluate their opinions of fidelity, factors that they felt may have influenced their fidelity and their views regarding elements that were useful and those that were less useful. Qualitative interview data on participants' views of the intervention will be obtained in individual, semi-structured interviews at intervention completion. We will use a version of the Consolidated Framework for Implementation Research optimised for use in Low and middle income countries (32) to conduct an implementation analysis that will inform the feasibility trial which will be finalised prior to a definitive trial.

Analysis will comprise qualitative and quantitative measures. Aggregate quantitative data will be analysed to determine intervention delivery feasibility. Descriptive statistics will be generated to assess attendance, attrition, retention, and the completeness of outcome measures. Pre-specified criterion for fidelity will be used interpreting $80-100 \%$ adherence as 'high' fidelity, $51-79 \%$ as 'moderate' and $0-50 \%$ as 'low' fidelity (33). Success criteria will be considered by the study team and the RAG at study completion. LMIC settings face unique challenges in delivering psychosocial interventions including lower levels of literacy, loss to follow-up arising from logistical issues such as inaccessible healthcare and residential transience and unavailability of caregivers to take part (34). The current evidence base is limited particularly in evaluating the delivery of psychosocial interventions by non-specialist healthcare workers. We will evaluate processes using semi-structured, qualitative interviews which will be digitally recorded, transcribed, checked for accuracy and analysed using framework analysis (35). Process evaluation will be informed by the Medical Research Council guidance on process evaluation in designing and testing interventions (36). Again, the interviews will be verified among the wider study team. Lastly. we will document our intervention according to the template for intervention description and replication (TIDieR) checklist (37).

\subsection{Training, Support and Supervision}

At the outset of the research project, we will deliver relevant elements of an adapted version of an existing research training programme developed by members of the study team for wider stakeholders and the research study team. Research training will be provided periodically, and training and support needs are continuously monitored. Research training will also be provided for researchers, advisors and stakeholders involved in our advisory group. Training in qualitative and quantitative data collection, principles of ethical research conduct, curation, data management and analysis is provided for research assistants. Trial therapists who will comprise healthcare workers in puskesmas will receive specialist training in family interventions which will be provided by a member of the research team who has significant expertise providing this training for NHS organisations. Supervision for intervention delivery will be provided by the qualified members of the study team. Research administration training for 
researchers in the feasibility study phase will be provided to include as before plus additional analysis, quantitative measurement and process evaluation.

\section{Discussion}

This study will provide important information on the feasibility of delivering family interventions for people with schizophrenia who reside in circumscribed areas of Indonesia. The intervention has a solid evidence base for delivery in high-income settings and is a NICE recommended intervention and the model of choice in NHS trusts $(23,38)$. Importantly, Family interventions have been successfully adapted to minority populations in the UK (39) and in other low resource settings $(31,40)$. As far as we are currently aware, these types of interventions have not previously been adapted culturally to Indonesian populations and contexts.

We have focused on implementation within the development phase of this research to ensure that Family interventions can be embedded within existing healthcare delivery settings using the more extensive network of primary care services available in Indonesia. Mental health treatment is often provided by mental health professionals whose names and qualifications are maintained in a central government register and they are licensed to practice in specialty settings. These specialist mental health providers are not readily available in Indonesia due to manpower and resource shortages, fledgling professional development, economically challenged populations and a lack of mental health as a priority in policy agendas. As an example, mental health nurses in Indonesia are not regulated by government legislation and degree courses have only been available since 2005. Our approach is guided by the World Health Organisations promotion of task shifting models to increase capacity for intervention delivery recognising the need for integration between primary and secondary mental health services and to develop family interventions that can be delivered by non-specialist professionals and are scalable.

Conducting a feasibility study presents multiple opportunities to refine procedures and processes to inform conduct of a more definitive trial at a later point. Through the intervention development stages data collected will inform intervention delivery during the feasibility study thus there are some aspects of how the study will run that are currently unknown including the format for delivery of the intervention, contents of the intervention and training needs of the healthcare workers. Similarly, there are key aspects of the feasibility study that are unknown including the endpoint of a future trial, instrumentation and study measures of feasibility. Our anticipated findings will be a testable, manualised intervention that aims to reduce relapse for people with psychosis and schizophrenia. We will be able to determine whether we can feasibly recruit sufficient participants to test the effects of the intervention. As before we are also interested in recruiting sufficient healthcare workers to determine whether they can be adequately trained to provide such an intervention in its intended format.

\section{Abbreviations}

NGO = non-governmental organisation 
KPSI = Komunitas Peduli Scizofreni

RAG = Research Advisory Group

RDMS = Research Data Management Service

$\mathrm{UI}=$ Universitas Indonesia

UoM = University of Manchester

\section{Declarations}

\section{Ethical Approval Statement}

Ethical approval has been granted by the ethical review committee at University of Manchester (Project ID 8041) and the equivalent at Universitas Indonesia. The project has been approved and permits supplied by the Ministry of Health in Indonesia. No data have been collected for this study as of submission date.

\section{Consent for publication}

No personal information provided in the manuscript

\section{Conflicts of Interest}

The authors report no conflicts of interest

\section{Data Availability Statement}

The authors confirm that data sharing is not applicable to this article as no new data were created or analysed in this study.

\section{CRediT Author Statement}

LR Conceptualisation, Funding acquisition, Methodology, Project administration, Supervision, Writing original draft, Writing -review \& editing, HS Conceptualisation, Funding acquisition, Methodology, Project administration, Supervision, Writing -review \& editing, HB Conceptualisation, Funding acquisition, Methodology, Project administration, Writing -review \& editing, BK Conceptualisation, Funding acquisition, Project administration, Supervision, Writing -review \& editing, TB Funding acquisition, Methodology, Project administration, Writing -review \& editing, PB Project administration, Resources, KL Conceptualisation, Funding acquisition, Methodology, Writing -review \& editing, KL Conceptualisation, Funding acquisition, Methodology, Writing -review \& editing

\section{Funding Sources}


This paper presents independent research funded by the Medical Research Council (MR/T003987/1) under its Joint Global Health Trials Funding Scheme titled 'Reducing Relapse for People with Schizophrenia in Jakarta, Indonesia: Developing a culturally-relevant, evidence-based Family Intervention'. Additional support was provided by the Faculty of Nursing at Universitas Indonesia. The views expressed are those of the authors and not necessarily those of the Medical Research Council, Universitas Indonesia or the University of Manchester.

\section{Acknowledgements}

We would like to acknowledge the invaluable assistance of Estin Yuliastuti, Raphita Diorarta and Rizky Fadilah in managing and coordinating research activity and providing resources for the study.

\section{References}

1. Whiteford HA, Degenhardt L, Rehm J, Baxter AJ, Ferrari AJ, Erskine HE, et al. Global burden of disease attributable to mental and substance use disorders: findings from the Global Burden of Disease Study 2010. The Lancet. 2014;382(9904):1575-86.

2. Charlson FJ, Ferrari AJ, Santomauro DF, Diminic S, Stockings E, Scott JG, et al. Global Epidemiology and Burden of Schizophrenia: Findings From the Global Burden of Disease Study 2016. Schizophr Bull. 2018;44(6):1195-203.

3. Lora A, Kohn R, Levav I, McBain R, Morris J, Saxena S. Service availability and utilization and treatment gap for schizophrenic disorders: a survey in 50 low- and middle-income countries. Bull World Health Organ. 2012;90(1):47-54. a-b.

4. Nuraini T, Tumanggor RD, Hungerford C, Lees D, Cleary M. Caregiver Burden for People with Schizophrenia in Medan, Indonesia. Issues Ment Health Nurs. 2020:1-4.

5. Annisa F. Burden of family caregiver. 2016. 2016;2(1):9.

6. Hidayat MT, Lawn S, Muir-Cochrane E, Oster C. The use of pasung for people with mental illness: a systematic review and narrative synthesis. International Journal of Mental Health Systems. 2020;14(1):90.

7. de Jesus Mari J, Razzouk D, Thara R, Eaton J, Thornicroft G. Packages of Care for Schizophrenia in Low- and Middle-Income Countries. PLoS Medicine. 2009;6(10):e1000165.

8. Patel V. Universal Health Coverage for Schizophrenia: A Global Mental Health Priority. Schizophr Bull. 2016;42(4):885-90.

9. Jamison DT, Gelband H, Horton S, Jha P, Laxminarayan R, Mock CN, et al. Disease Control Priorities, Third Edition: Volume 9. Improving Health and Reducing Poverty. Washington, DC: World Bank. Washington D.C.: World Bank;; 2017.

10. Pharoah F, Mari J, Rathbone J, Wong W. Family intervention for schizophrenia. The Cochrane database of systematic reviews. 2010(12):CD000088-CD. 
11. Sin J, Gillard S, Spain D, Cornelius V, Chen T, Henderson C. Effectiveness of psychoeducational interventions for family carers of people with psychosis: A systematic review and meta-analysis. Clin Psychol Rev. 2017;56:13-24.

12. Asher L, Patel V, De Silva MJ. Community-based psychosocial interventions for people with schizophrenia in low and middle-income countries: systematic review and meta-analysis. BMC Psychiatry. 2017;17(1):355.

13. Pharoah F, Mari J, Rathbone J, Wong W. Family intervention for schizophrenia. The Cochrane database of systematic reviews. 2006;18(4).

14. Viana MC, Gruber MJ, Shahly V, Alhamzawi A, Alonso J, Andrade LH, et al. Family burden related to mental and physical disorders in the world: results from the WHO World Mental Health (WMH) surveys. Brazilian Journal of Psychiatry. 2013;35:115-25.

15. Stanley S, Balakrishnan S, Ilangovan S. Psychological distress, perceived burden and quality of life in caregivers of persons with schizophrenia. Journal of Mental Health. 2017;26(2):134-41.

16. Pitschel-Walz G, Leucht S, Bauml J, Kissling W, Engel RR. The effect of family interventions on relapse and rehospitalization in schizophrenia-a meta-analysis. Schizophr Bull. 2001;27(1):73-92.

17. Claxton M, Onwumere J, Fornells-Ambrojo M. Do Family Interventions Improve Outcomes in Early Psychosis? A Systematic Review and Meta-Analysis. Frontiers in psychology. 2017;8:371-.

18. Bird V, Premkumar P, Kendall T, Whittington C, Mitchell J, Kuipers E. Early intervention services, cognitive-behavioural therapy and family intervention in early psychosis: systematic review. The British Journal of Psychiatry. 2010;197(5):350-6.

19. Degnan A, Baker S, Edge D, Nottidge W, Noke M, Press CJ, et al. The nature and efficacy of culturallyadapted psychosocial interventions for schizophrenia: a systematic review and meta-analysis. Psychological medicine. 2017;48(5):714-27.

20. Joshi R, Alim M, Kengne AP, Jan S, Maulik PK, Peiris D, et al. Task Shifting for Non-Communicable Disease Management in Low and Middle Income Countries - A Systematic Review. PLOS ONE. 2014;9(8):e103754.

21. Seidman G, Atun R. Does task shifting yield cost savings and improve efficiency for health systems? A systematic review of evidence from low-income and middle-income countries. Human Resources for Health. 2017;15(1):29.

22. Craig P, Dieppe P, Macintyre S, Michie S, Nazareth I, Petticrew M. Developing and evaluating complex interventions: The new Medical Research Council guidance. International journal of nursing studies. 2013;50(5):587-92.

23. Barrowclough C, Tarrier N. Families of schizophrenic patients: cognitive behavioural intervention. London: Chapman \& Hall; 1992.

24. Idaiani S, Yunita I, Tjandrarini D, Indrawati L, Darmayanti I, Kusumawardani N, et al. Prevalensi Psikosis di Indonesia berdasarkan Riset Kesehatan Dasar 2018. Jurnal Penelitian Dan Pengembangan Pelayanan Kesehatan. 2019;3:9-16. 
25. Menteri Kesehetan Republik Indonesia. STANDAR TARIF PELAYANAN KESEHATAN DALAM PENYELENGGARAAN PROGRAM JAMINAN KESEHATAN. In. Indonesia MKR, editor. 2016.

26. Heim E, Shehadeh MH, van't Hof E, Carswell K. Cultural adaptation of scalable interventions. Cultural clinical psychology and PTSD. Boston: Hogrefe Publishing; 2019. pp. 201-18.

27. Raine R, Sanderson C, Hutchings A, Carter S, Larkin K, Black N. An experimental study of determinants of group judgments in clinical guideline development. Lancet. 2004;364(9432):42937.

28. Larkin M, Boden ZVR, Newton E. On the Brink of Genuinely Collaborative Care: Experience-Based CoDesign in Mental Health. Qual Health Res. 2015;25(11):1463-76.

29. Braun V, Clarke V. Using thematic analysis in psychology. Qualitative research in psychology. 2006;3(2):77-101.

30. Twinn S. An exploratory study examining the influence of translation on the validity and reliability of qualitative data in nursing research. J Adv Nurs. 1997;26(2):418-23.

31. Rathod S, Gega L, Degnan A, Pikard J, Khan T, Husain N, et al. The current status of culturally adapted mental health interventions: a practice-focused review of meta-analyses. Neuropsychiatr Dis Treat. 2018;14:165-78.

32. Means AR, Kemp CG, Gwayi-Chore M-C, Gimbel S, Soi C, Sherr K, et al. Evaluating and optimizing the consolidated framework for implementation research (CFIR) for use in low- and middle-income countries: a systematic review. Implementation Science. 2020;15(1):17.

33. Borrelli B, Sepinwall D, Ernst D, Bellg AJ, Czajkowski S, Breger R, et al. A new tool to assess treatment fidelity and evaluation of treatment fidelity across 10 years of health behavior research. $J$ Consult Clin Psychol. 2005;73(5):852-60.

34. Brooke-Sumner C, Petersen I, Asher L, Mall S, Egbe CO, Lund C. Systematic review of feasibility and acceptability of psychosocial interventions for schizophrenia in low and middle income countries. BMC Psychiatry. 2015;15(1):19.

35. Ritchie J, Lewis J, McNaughton Nicholls C, Ormston R. Qualitative research practice: a guide for social science students and researchers2014.

36. Moore GF, Audrey S, Barker M, Bond L, Bonell C, Hardeman W, et al. Process evaluation of complex interventions: Medical Research Council guidance. BMJ: British Medical Journal. 2015;350:h1258.

37. Hoffmann TC, Glasziou PP, Boutron I, Milne R, Perera R, Moher D, et al. Better reporting of interventions: template for intervention description and replication (TIDieR) checklist and guide2014 2014-03-07 10:29:06.

38. National Institute for Health and Care Excellence (NICE). Psychosis and schizophrenia in adults: treatment and management: CG178. London: National Institute for Health and Clinical Excellence; 2014.

39. Edge D, Degnan A, Cotterill S, Berry K, Drake R, Baker J, et al. Culturally-adapted Family Intervention (CaFI) for African-Caribbeans diagnosed with schizophrenia and their families: a feasibility study protocol of implementation and acceptability. Pilot Feasibility Studies. 2016;2(1):39. 
40. Husain MO, Khoso AB, Renwick L, Kiran T, Saeed S, Lane S, et al. Culturally adapted family intervention for schizophrenia in Pakistan: a feasibility study. Int J Psychiatry Clin Pract. 2020:1-10.

\section{Tables}

Due to technical limitations, table 1,2,3 is only available as a download in the Supplemental Files section.

\section{Supplementary Files}

This is a list of supplementary files associated with this preprint. Click to download.

- Table1.jpg

- Table2.jpg

- Table3.jpg

- SPIRITChecklist.doc 